\title{
Expression of Glycolysis-Related Proteins in Cancer of Unknown Primary Origin
}

\author{
Murilo Bonatelli ${ }^{1}$, Isabella Fernandes Fornari ${ }^{1,2}$, Priscila Neves Bernécule ${ }^{1,2}$, \\ Lara Esquiapatti Pinheiro ${ }^{1,2}$, Ricardo Filipe Alves Costa ${ }^{2,3}$, \\ Adhemar Longatto-Filho ${ }^{1,4,5,6}$, João Neif Antonio Junior ${ }^{7}$, \\ Eduardo Caetano Albino Silva ${ }^{8}$, Flávio Mavignier Cárcano ${ }^{2,7}$ and Céline Pinheiro ${ }^{1,2 *}$ \\ ${ }^{1}$ Molecular Oncology Research Center, Barretos Cancer Hospital, Barretos, Brazil, ${ }^{2}$ Barretos School of Health Sciences \\ Dr. Paulo Prata-FACISB, Barretos, Brazil, ${ }^{3}$ Research and Teaching Institute, Barretos Cancer Hospital, Barretos, Brazil, \\ ${ }^{4}$ Life and Health Sciences Research Institute (ICVS), School of Medicine, University of Minho, Braga, Portugal, 5 ICVS/3B's \\ PT Government Associate Laboratory, Braga, Portugal, ${ }^{6}$ Laboratory of Medical Investigation (LIM-14), School of Medicine, \\ University of São Paulo, São Paulo, Brazil, ${ }^{7}$ Medical Oncology Department, Barretos Cancer Hospital, Barretos, Brazil, \\ ${ }^{8}$ Pathology Department, Barretos Cancer Hospital, Barretos, Brazil
}

\section{OPEN ACCESS}

Edited by: Maria Ida Amabile,

Sapienza University of Rome, Italy

Reviewed by:

Anne Devin,

Centre National de la Recherche Scientifique (CNRS), France

Jiri Neuzil,

Griffith University, Australia

*Correspondence:

Céline Pinheiro

celinepinheiro@gmail.com

Specialty section:

This article was submitted to

Cancer Metabolism

a section of the journal

Frontiers in Oncology

Received: 18 March 2021 Accepted: 07 June 2021

Published: 24 June 2021

Citation:

Bonatelli M, Fornari IF, Bernécule PN, Pinheiro LE, Costa RFA, Longatto-Filho A, Junior JNA, Silva ECA, Cárcano FM and Pinheiro C (2021) Expression of Glycolysis-Related Proteins in Cancer of Unknown Primary Origin.

Front. Oncol. 11:682665.

doi: 10.3389/fonc.2021.682665
Introduction: Cancer of unknown primary origin (CUP) is defined as metastatic cancer without identification of the primary site. Considering that only $15-20 \%$ of patients with CUP show a favorable outcome, identifying biomarkers may help improve the clinical management of patients who do not respond well to conventional therapies. In this context, the study of the metabolic profile of CUP may pave the way to establish new biomarkers and/or therapeutic targets; therefore, this study aimed to characterize the expression of metabolism-related proteins in CUP.

Materials and Methods: The expression of monocarboxylate transporters MCT1, MCT2 and MCT4, their chaperone CD147, the glucose transporter GLUT1 and the pH regulator CAIX was evaluated by immunohistochemistry in a series of 118 CUP patients, and the results were associated with the available clinicopathological information.

Results: The metabolism-related proteins MCT1, MCT4, CD147, GLUT1 and CAIX were expressed in a critical portion of the CUP (approximately 20 to 70\%). MCT1 and CD147 were both more frequently expressed in cases with lymph nodes as metastasis dominant sites $(p=0.001)$ as well as in samples from lymph nodes $(p<0.001$ and $p=0.002$, respectively), while MCT1 expression was more frequently expressed in squamous cell carcinomas $(p=0.045)$. A higher overall survival was observed in patients with tumors positive for GLUT1 and CAIX expression ( $p=0.011$ and $p=0.041$, respectively), but none of the proteins was an independent prognostic factor for overall survival in multivariable analysis.

Conclusion: The results suggest that a portion of CUPs present a hyperglycolytic phenotype, which is associated with higher overall survival.

Keywords: immunohistochemistry, glycolytic metabolism, metabolic reprogramming, Warburg effect, cancer of unknown primary origin 


\section{INTRODUCTION}

Cancer of unknown primary origin (CUP) is defined as metastatic cancer without identification of the primary site, even after careful evaluation (1). In the early 1990s, CUP accounted for 3-5\% of all cancer diagnoses; however, advances in radiological and molecular aspects have improved the identification of primary tumor sites, decreasing the diagnostic rate of CUP to $1-2 \%(2,3)$. The investigation of this tumor type includes a detailed physical examination, laboratory tests, histopathological review of the material used for immunohistochemistry, and pelvic, thorax and abdominal computed tomography (1). CUP has a very heterogeneous clinical presentation, unpredictable metastatic spread, high aggressiveness and a low response to chemotherapy, presenting very low survival, with only $15-20 \%$ of patients showing a favorable outcome (4). For patients involved in clinical trials, the median survival is $6-10$ months, and fewer than $25 \%$ of patients survive more than one year. For those who are not enrolled in clinical trials, the survival rates decrease to $2-3$ months (5). Importantly, despite advances in the diagnosis and therapy fields for CUP patients, the mechanisms of carcinogenesis, development and progression of these tumors remain unclear $(3,6)$.

Metabolic rewiring in tumors is gaining increased attention and is a hallmark of cancer (7). This phenomenon has been described as the Warburg effect or aerobic glycolysis $(8,9)$, in which tumor cells preferentially produce energy through glycolysis rather than mitochondrial oxidative phosphorylation (10). Although aerobic glycolysis is far less efficient than oxidative phosphorylation for ATP production, this metabolic adaptation is required to use other carbon source pathways as building blocks for biomolecule syntheses, which are essential for cell proliferation. The accumulation of these important portions of carbons as glycolytic intermediates feeds anabolic pathways, producing proteins, lipids and nucleic acids and maintaining an increased state of cellular proliferation, which favors tumor cell survival over normal cells $(11,12)$. Furthermore, the loss of a combination of tumor suppressor genes, such as TP53, and activation of oncogenes (oncogene addiction), particularly related to the PI3K-AKT axis, which is linked to both growth control and glucose metabolism, are intrinsic tumor mechanisms in the orchestration of metabolic reprogramming (11). Several proteins play an important role in the metabolic reprogramming of tumor cells (13), such as glucose transporter 1 (GLUT1), a transmembrane protein that facilitates glucose influx $(14,15)$, monocarboxylate transporters (MCTs), particularly isoforms 1 and 4, because of their function in lactate efflux (16) in close association with their chaperone CD147 (17), and carbonic anhydrase 9 (CAIX), a $\mathrm{pH}$ regulator contributing to preservation of intracellular $\mathrm{pH}$ (18). Therefore, these proteins have increased expression and are potential therapeutic targets for cancer treatment $(13,16,19)$.

A recent study in CUP has shown differences in the expression profile of proteins related to metabolism, according to the histological subtype and clinical characteristics (20); however, additional studies are warranted to provide robust information on the metabolic characteristics of CUP. The identification of prognostic markers may contribute to the treatment of CUP patients who do not respond well to conventional therapies, providing alternatives to the treatment employed. In this context, the study of the metabolic profile of tumor cells may pave the way to establish new markers and/or therapeutic targets (21). Thus, this study aimed to characterize the expression of MCT1, MCT2, MCT4, CD147, GLUT1 and CAIX in the CUP.

\section{MATERIALS AND METHODS}

\section{Case Selection and Clinicopathological Information}

The study series included 118 CUP patients diagnosed from 2002 to 2017 at the Barretos Cancer Hospital. The inclusion criteria used were clinical/radiological CUP diagnoses and an immunohistochemical panel according to the discretion of the pathologist (more frequently evaluated markers: cytokeratin 7 , cytokeratin 20, thyroid transcription factor-1, vimentin and carcinoembryonic antigen), PSA (prostate-specific antigen) evaluation in men, HCG (human chorionic gonadotropin) evaluation in poorly differentiated carcinomas cases and formalin-fixed paraffin-embedded tissues available at the Pathology Department. Patients were excluded by the following criteria: poorly differentiated squamous cell carcinoma limited to cervical lymph nodes, women with adenocarcinoma limited to axillary lymph nodes, women with adenocarcinoma and involvement of the peritoneal cavity only, young men $(<55$ years) with middle line tumor growth, neuroendocrine carcinoma, melanoma metastasis, the presence of other primary malignancies (except non-melanoma tumors), positive HIV serology and patients on chronic use of immunosuppressive agents.

Sociodemographic and clinicopathological features included, among others, the age at diagnosis, sex, lifestyle habits (smoking and alcoholism status), tumor histological subtype, first-line treatment and follow-up. The detailed data are presented in Table 1. The study data were collected and managed using REDCap electronic data capture tools hosted at the Barretos Cancer Hospital $(23,24)$. The Barretos Cancer Hospital Ethics Committee approved the present study (1055/2015, CAAE 51579715.0.0000.5437).

\section{Immunohistochemistry}

MCT1, MCT2, MCT4, CD147, GLUT1 and CAIX expression was evaluated by immunohistochemistry in histological sections of cancer of unknown primary origin samples. Detailed information on antigen retrieval and each antibody used are described in Table 2. Immunohistochemistry for MCT1 and CD147 was performed using a polymer system (Ultra Vision ONE Detection System: HRP Polymer, Lab Vision Corporation, Fremont, CA) as previously described (25). MCT4 reactions were performed using a streptavidin-biotin-peroxidase complex (Ultravision Detection System: Large Volume Anti-Polyvalent, HRP, Lab Vision Corporation, Fremont, CA), as previously described (26); finally, MCT2, GLUT1 and CAIX reactions were performed using a biotin-free principle (ADVANCE HRP; Dako, Carpinteria, CA) according to the manufacturer's 
TABLE 1 | Sociodemographic and clinicopathological characteristics of patients with cancer of unknown primary origin.

\begin{tabular}{|c|c|}
\hline Sociodemographic and clinicopathological characteristics & n (\%) \\
\hline All patients & $118(100.0)$ \\
\hline \multicolumn{2}{|l|}{ Age $($ Mean \pm SD $=59.5 \pm 12.7$ Min-Max $=19.9-88.8)$} \\
\hline$<59.5$ years & $57(48.3)$ \\
\hline$\geq 59.5$ years & $61(51.7)$ \\
\hline \multicolumn{2}{|l|}{ Sex } \\
\hline Female & $63(53.4)$ \\
\hline Male & $55(46.6)$ \\
\hline \multicolumn{2}{|l|}{ Smoker } \\
\hline Yes & $50(42.4)$ \\
\hline No & $62(52.5)$ \\
\hline Unknown & $6(5.0)$ \\
\hline \multicolumn{2}{|l|}{ Alcoholism } \\
\hline Yes & $25(21.2)$ \\
\hline No & $81(68.6)$ \\
\hline Unknown & $12(10.1)$ \\
\hline \multicolumn{2}{|l|}{ Cancer family history } \\
\hline Yes & $44(37.2)$ \\
\hline No & $59(50.0)$ \\
\hline Unknown & $15(12.7)$ \\
\hline \multicolumn{2}{|l|}{ Metastasis dominant site } \\
\hline Liver & $45(52.4)$ \\
\hline Bone & $30(38.1)$ \\
\hline Lymph node & $27(22.9)$ \\
\hline Lung & $3(2.5)$ \\
\hline Central nervous system & $2(1.7)$ \\
\hline Others & $11(9.3)$ \\
\hline \multicolumn{2}{|l|}{ Biopsy site } \\
\hline Liver & $40(33.9)$ \\
\hline Bone & $27(22.9)$ \\
\hline Lymph node & $31(26.2)$ \\
\hline Central nervous system & $2(1.7)$ \\
\hline Others & $15(12.7)$ \\
\hline Unknown & $3(2.5)$ \\
\hline \multicolumn{2}{|l|}{ Histological subtype } \\
\hline Adenocarcinoma & $67(56.8)$ \\
\hline Carcinoma & $13(11.0)$ \\
\hline Squamous cell carcinoma & $9(7.6)$ \\
\hline Well-differentiated adenocarcinoma & $1(0.8)$ \\
\hline Poorly differentiated adenocarcinoma & $8(6.8)$ \\
\hline Poorly differentiated carcinoma & $16(13.5)$ \\
\hline Not classified & $4(3.4)$ \\
\hline \multicolumn{2}{|l|}{ PS (ECOG) } \\
\hline $0-1$ & $48(40.7)$ \\
\hline$\geq 2$ & 35 (29.6) \\
\hline Unknown & 35 (29.6) \\
\hline \multicolumn{2}{|l|}{ First-Line Chemotherapy } \\
\hline Etoposide/Cisplatin & $1(0.8)$ \\
\hline Carboplatin/Paclitaxel & $20(16.9)$ \\
\hline Gemcitabine/Cisplatin & $5(4.2)$ \\
\hline Others & $25(21.2)$ \\
\hline No Chemotherapy & $67(56.7)$ \\
\hline
\end{tabular}

$\overline{S D}$, standard deviation; PS (ECOG), performance status (Eastern Cooperative Oncology Group) (22).

instructions and as previously described (25). For visualization, the slides were incubated with 3,3'-diaminobenzidine (Liquid $\mathrm{DAB}+$ Substrate Chromogen System; Dako, Carpinteria, CA) according to the manufacturer's instructions, counterstained with hematoxylin and permanently mounted. As positive controls, squamous cell carcinoma of the oral cavity was used for MCT1 and MCT4, normal kidney for MCT2, normal colon for CD147, placenta for GLUT1 and normal gastric mucosa for CAIX. Negative controls were available in the same tissue sections used as positive controls.

\section{Immunohistochemical Evaluation}

The reactions were scored semi-quantitatively for the extension of expression in cancer cells as follows: 0: no immunoreactive cells; 1 : $<5 \%$ immunoreactive cells; 2 : $5-50 \%$ immunoreactive cells; and 3: $>50 \%$ immunoreactive cells. Additionally, the intensity of staining was scored semi-qualitatively as follows: 0 : negative; 1 : weak; 2 : intermediate; and 3 : strong. The final score was defined as the sum of both parameters (extension and intensity) and grouped as negative (scores $0-2$ ) and positive (score 3-6), as previously described (27). Only protein expression in the plasma membrane was considered for further analysis. The reactions were evaluated by two pathologists (ECAS and AL).

\section{Statistical Analysis}

The data collected were analyzed using the Statistical Package for Social Sciences (SPSS) software for Windows (version 21.0). Statistical significance for comparisons between biomarker immunoexpression and clinicopathological parameters, as well as co-expression analysis, were evaluated using Pearson's chi-squared $\left(\chi^{2}\right)$ and Fisher's exact tests, according to the series characteristics. Kaplan-Meier analysis was used to assess overall survival, and comparisons between survival curves were performed using logrank tests. The time to event of interest considered was the date of diagnosis until the date of death. Multivariable analysis of survival was performed using the Cox proportional hazards regression model. Independent variables were analyzed by univariable analysis, followed by multivariable analysis of all variables that reached a $\mathrm{p}$-value $<0.100$ at univariable analysis. The threshold of significant $p$ values was established as $p<0.050$.

\section{RESULTS}

\section{Expression of MCT1, MCT2, MCT4, CD147, GLUT1 and CAIX in Cancer of Unknown Primary Origin Patients}

Immunohistochemical expression of metabolism-related proteins in CUP patients (Figure 1) showed that the expression of MCT4, CD147, GLUT1 and CAIX was mostly found in the plasma membrane (approximately $80 \%$ of positive samples). For MCT1 and MCT2, plasma membrane expression was found in 52.3 and $33.3 \%$ of positive cases, respectively. Considering only plasma membrane expression, MCT1 was found in $23(19.5 \%)$, MCT2 in three $(2.5 \%)$, MCT4 in 79 (66.9\%), CD147 in 40 (33.9\%), GLUT1 in $53(44.9 \%)$ and CAIX in $40(33.9 \%)$ CUP samples.

\section{Clinicopathological Significance of MCTs, CD147, GLUT1 and CAIX in Cancer of Unknown Primary Origin Patients}

The associations between the clinicopathological data and expression of metabolism-related proteins in CUP patients are 
TABLE 2 | Detailed aspects of immunohistochemistry.

\begin{tabular}{|c|c|c|c|c|}
\hline Protein & Antigen retrieval & Antibody & Clonality & Dilution, incubation time and temperature \\
\hline MCT1 & EDTA (1 mM, pH = 8.0), $98^{\circ} \mathrm{C}, 20$ & $\begin{array}{l}\text { AB3538P } \\
\text { Chemicon International }\end{array}$ & Polyclonal & 1:300, overnight, RT \\
\hline MCT2 & Citrate $(0.01 \mathrm{M}, \mathrm{pH}=6.0), 98^{\circ} \mathrm{C}, 20 \mathrm{~min}$ & $\begin{array}{l}\text { sc-50322 } \\
\text { Santa Cruz Biotechnology }\end{array}$ & Polyclonal & $1: 800,2$ h, RT \\
\hline MCT4 & Citrate $(0.01 \mathrm{M}, \mathrm{pH}=6.0), 98^{\circ} \mathrm{C}, 20 \mathrm{~min}$ & $\begin{array}{l}\text { sc-50329 } \\
\text { Santa Cruz Biotechnology }\end{array}$ & Polyclonal & 1:500, 2 h, RT \\
\hline CD147 & EDTA (1 mM, pH = 8.0), $98^{\circ} \mathrm{C}, 20 \mathrm{~min}$ & $\begin{array}{l}\text { sc-71038 } \\
\text { Santa Cruz Biotechnology }\end{array}$ & Monoclonal (1.BB.218) & 1:500, overnight, $R T$ \\
\hline GLUT1 & Citrate $(0.01 \mathrm{M}, \mathrm{pH}=6.0), 98^{\circ} \mathrm{C}, 20 \mathrm{~min}$ & $\begin{array}{l}\text { ab15309 } \\
\text { Abcam }\end{array}$ & Polyclonal & 1:500, 2 h, RT \\
\hline CAIX & Citrate $(0.01 \mathrm{M}, \mathrm{pH}=6.0), 98^{\circ} \mathrm{C}, 20 \mathrm{~min}$ & $\begin{array}{l}\text { ab15086 } \\
\text { Abcam }\end{array}$ & Polyclonal & 1:4,000, 2 h, RT \\
\hline
\end{tabular}

$R T$, room temperature.

shown in Table 3. MCT1 expression was more frequently expressed in cases with lymph nodes as the metastasis dominant site $(p=0.001)$, as well as in biopsies from lymph nodes $(p<0.001)$ and in squamous cell carcinomas $(p=0.045)$. The MCT chaperone CD147 was significantly associated with the metastasis dominant site $(p=0.001)$ and biopsy site $(p=0.002)$, following the same frequency pattern of MCT1. MCT2 (data not shown because of a low number of positive cases), MCT4, CD147, GLUT1 and CAIX showed no significant associations with the clinicopathological data.

\section{Co-Expression Analysis}

When evaluating the co-expression of MCTs with the other metabolism-related proteins in CUP patients (Table 4), we found that MCT1 and MCT4 were significantly co-expressed with CD147 ( $p=0.001$ and $p<0.001$, respectively). Additionally,
MCT4 showed significant co-expression with both GLUT1 ( $p=$ $0.006)$ and CAIX $(p=0.036)$.

Regarding the association of the glycolytic phenotype and clinicopathological parameters of CUP patients (Table 5), we found that MCT1 and CD147, as well as MCT4 and CD147 coexpression, were more frequently found in cases with lymph nodes as the metastasis dominant site $(p<0.001)$ and biopsies from lymph nodes $(p<0.001)$.

\section{Survival Analysis}

Overall survival analysis of MCTs, CD147, GLUT1 and CAIX in CUP patients (Figure 2) showed that positive GLUT1 and CAIX expression was significantly associated with higher overall survival ( $p=0.011$ and $p=0.041$, respectively). Regarding the influence of co-expression with the overall survival of CUP patients,

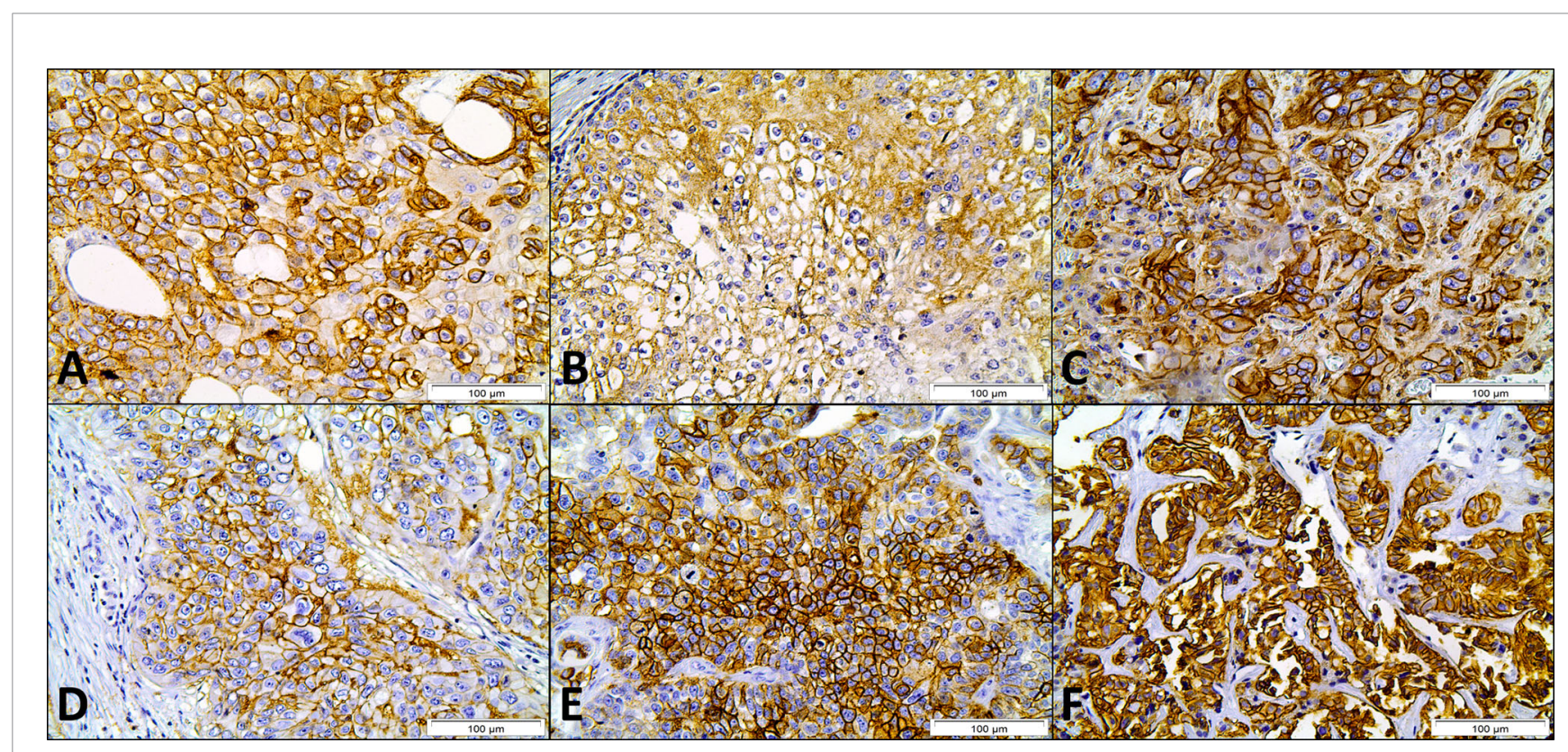

FIGURE 1 | Immunohistochemical expression of MCT1 (A), MCT2 (B), MCT4 (C), CD147 (D), GLUT1 (E) and CAIX (F) in cancer of unknown primary origin samples. Magnification: 400x. 
TABLE 3 | Association of MCT1, MCT4, CD147, GLUT1 and CAIX membrane expression with the clinicopathological characteristics of cancer of unknown primary origin patients.

\begin{tabular}{|c|c|c|c|c|c|c|c|c|c|c|c|c|c|c|c|}
\hline & \multicolumn{3}{|c|}{ MCT1 } & \multicolumn{3}{|c|}{ MCT4 } & \multicolumn{3}{|c|}{ CD147 } & \multicolumn{3}{|c|}{ GLUT1 } & \multicolumn{3}{|c|}{ CAIX } \\
\hline$<59.7$ & 54 & $12(22.2)$ & & 56 & 41 (73.2) & & 53 & $17(32.1)$ & & 52 & $22(42.3)$ & & 50 & $18(36.0)$ & \\
\hline$\geq 59.7$ & 60 & 11 (18.3) & & 59 & $38(64.4)$ & & 58 & $23(39.7)$ & & 58 & $31(53.4)$ & & 57 & 22 (38.6) & \\
\hline Sex & & & 0.812 & & & 0.716 & & & 0.617 & & & 0.718 & & & 0.083 \\
\hline Smoker & & & 0.557 & & & 0.138 & & & 0.379 & & & 0.826 & & & 0.858 \\
\hline No & 60 & $11(18.3)$ & & 61 & 39 (63.9) & & 57 & 19 (33.3) & & 55 & $27(49.1)$ & & 52 & $20(38.5)$ & \\
\hline Yes & 48 & $11(22.9)$ & & 48 & $37(77.1)$ & & 48 & $20(41.7)$ & & 49 & $23(46.9)$ & & 49 & $18(36.7)$ & \\
\hline Alcoholism & & & 0.459 & & & 0.910 & & & 0.933 & & & 0.908 & & & 0.116 \\
\hline No & 79 & 15 (19.0) & & 79 & 55 (69.6) & & 76 & 29 (38.2) & & 74 & 36 (48.6) & & 73 & 29 (39.7) & \\
\hline Yes & 23 & $6(26.1)$ & & 24 & $17(70.8)$ & & 23 & 9 (39.1) & & 24 & $12(50.0)$ & & 23 & $5(21.7)$ & \\
\hline Liver & 42 & $5(11.9)$ & & 42 & 26 (61.9) & & 41 & $9(22.0)$ & & 41 & $20(48.8)$ & & 41 & 15 (36.6) & \\
\hline Bone & 30 & $2(6.7)$ & & 30 & 19 (63.3) & & 28 & $7(25.0)$ & & 27 & 9 (33.3) & & 26 & 7 (26.9) & \\
\hline Lymph node & 27 & $11(40.7)$ & & 27 & $21(77.8)$ & & 27 & 17 (63.0) & & 27 & 15 (55.6) & & 26 & $11(42.3)$ & \\
\hline Biopsy site & & & $<0.001$ & & & 0.527 & & & 0.002 & & & 0.311 & & & 0.660 \\
\hline Liver & 38 & $3(7.9)$ & & 37 & $23(62.2)$ & & 36 & $6(16.7)$ & & 37 & 19 (51.4) & & 35 & $13(37.1)$ & \\
\hline Bone & 27 & $2(7.4)$ & & 27 & $17(63.0)$ & & 25 & 8 (32.0) & & 24 & 8 (33.3) & & 25 & $7(28.0)$ & \\
\hline Lymph node & 30 & $14(46.7)$ & & 31 & $23(74.2)$ & & 31 & $18(58.1)$ & & 29 & $15(51.7)$ & & 28 & $11(39.3)$ & \\
\hline Histological subtype & & & 0.045 & & & 0.972 & & & 0.084 & & & 0.748 & & & 0.738 \\
\hline Adenocarcinoma & 65 & $9(13.8)$ & & 65 & 45 (69.2) & & 62 & 17 (27.4) & & 61 & 27 (44.3) & & 60 & $25(41.7)$ & \\
\hline Carcinoma & 13 & 5 (38.5) & & 12 & $8(66.7)$ & & 12 & $5(41.7)$ & & 13 & $7(53.8)$ & & 12 & $3(25.0)$ & \\
\hline Squamous cell carcinoma & 8 & $4(50.0)$ & & 9 & $7(77.8)$ & & 9 & $6(66.7)$ & & 9 & $6(66.7)$ & & 9 & $3(33.3)$ & \\
\hline Poorly differentiated & 8 & $1(12.5)$ & & 8 & $5(62.5)$ & & 8 & $2(25.0)$ & & 8 & $4(50.0)$ & & 7 & $2(28.6)$ & \\
\hline adenocarcinoma & & & & & & & & & & & & & & & \\
\hline
\end{tabular}

PS (ECOG), performance status (Eastern Cooperative Oncology Group) (22). *Fisher's exact test. Significant p-values are depicted in bold.

MCT1 and CD147, when positively co-expressed, showed better overall survival rates $(p=0.042)$, as well as MCT4/GLUT1 $(p=$ $0.030)$ and MCT4/CAIX ( $p=0.016)$ co-expression.

The prognostic values of the proteins and clinicopathological parameters for overall survival were assessed using Cox proportional hazards regression models (Table 6). Univariable analysis revealed the prognostic values of the performance status and CAIX expression. Multivariable analysis showed that a performance status $\geq 2$ was an independent prognostic factor for overall survival (HR: 2.352, $p=0.002$ ). None of the analyzed metabolism-related proteins presented a prognostic value in the multivariable analysis of CUP patients.

TABLE 4 | Co-expression of MCTs with CD147, GLUT1 and CAIX in cancer of unknown primary origin patients.

\begin{tabular}{|c|c|c|c|c|c|c|}
\hline & \multicolumn{3}{|c|}{ MCT1 } & \multicolumn{3}{|c|}{ MCT4 } \\
\hline CD147 & & & 0.001 & & & $<0.001$ \\
\hline Positive & 38 & 15 (39.5) & & 40 & $36(90.0)$ & \\
\hline GLUT1 & & & 0.421 & & & 0.006 \\
\hline Negative & 55 & 10 (18.2) & & 56 & 33 (58.9) & \\
\hline Negative & 65 & 14 (21.5) & & 67 & $42(62.7)$ & \\
\hline Positive & 40 & $9(22.5)$ & & 39 & 32 (82.1) & \\
\hline
\end{tabular}

Significant $p$-values are depicted in bold. 
TABLE 5 | Co-expression association of metabolism-related proteins with the clinicopathological characteristics of cancer of unknown primary origin patients.

\begin{tabular}{|c|c|c|c|c|c|c|c|c|c|c|c|c|}
\hline & \multicolumn{3}{|c|}{ MCT1/CD147 } & \multicolumn{3}{|c|}{ MCT4/CD147 } & \multicolumn{3}{|c|}{ MCT4/GLUT1 } & \multicolumn{3}{|c|}{ MCT4/CAIX } \\
\hline & $\mathbf{n}$ & Positive (\%) & $p$ & $\mathbf{n}$ & Positive (\%) & $p$ & $\mathbf{n}$ & Positive (\%) & $p$ & $\mathbf{n}$ & Positive (\%) & $p$ \\
\hline Age (years) & & & 0.923 & & & 0.627 & & & 0.273 & & & 0.957 \\
\hline$<59.7$ & 55 & $7(12.7)$ & & 54 & 16 (29.6) & & 54 & 18 (33.3) & & 52 & 15 (28.8) & \\
\hline$\geq 59.7$ & 60 & 8 (13.3) & & 59 & $20(33.9)$ & & 60 & 26 (43.3) & & 58 & 17 (29.3) & \\
\hline Sex & & & 0.663 & & & 0.324 & & & 0.860 & & & 0.439 \\
\hline Female & 52 & $6(11.5)$ & & 52 & $19(36.5)$ & & 53 & $20(37.7)$ & & 51 & $13(25.5)$ & \\
\hline Male & 63 & 9 (14.3) & & 61 & $17(27.9)$ & & 61 & 24 (39.3) & & 59 & $19(32.2)$ & \\
\hline Smoker & & & 0.735 & & & 0.172 & & & 0.441 & & & 0.247 \\
\hline No & 61 & $9(14.8)$ & & 59 & $16(27.1)$ & & 59 & 21 (35.6) & & 56 & $14(25)$ & \\
\hline Yes & 48 & $6(12.5)$ & & 48 & 19 (39.6) & & 49 & $21(42.9)$ & & 48 & 17 (35.4) & \\
\hline Alcoholism & & & $1.000^{\star}$ & & & 0.897 & & & 0.867 & & & 0.476 \\
\hline No & 80 & $12(15.0)$ & & 78 & 26 (33.3) & & 78 & $31(39.7)$ & & 75 & 22 (29.3) & \\
\hline Yes & 23 & $3(13.0)$ & & 23 & 8 (34.8) & & 24 & $10(41.7)$ & & 23 & $5(21.7)$ & \\
\hline Cancer family history & & & 0.932 & & & 0.901 & & & 0.432 & & & 0.677 \\
\hline No & 59 & 9 (15.3) & & 56 & $18(32.1)$ & & 57 & $20(35.1)$ & & 54 & $15(27.8)$ & \\
\hline Yes & 41 & $6(14.6)$ & & 42 & $14(33.3)$ & & 42 & $18(42.9)$ & & 41 & $13(31.7)$ & \\
\hline Metastasis dominant site & & & $<0.001$ & & & $<0.001$ & & & 0.418 & & & 0.142 \\
\hline Liver & 42 & $2(4.8)$ & & 42 & $7(16.7)$ & & 43 & $15(34.9)$ & & 41 & $10(24.4)$ & \\
\hline Bone & 30 & 1 (3.3) & & 29 & $6(20.7)$ & & 29 & $8(27.6)$ & & 28 & $5(17.9)$ & \\
\hline Lymph node & 27 & 9 (33.3) & & 27 & $16(59.3)$ & & 27 & $12(44.4)$ & & 27 & $11(40.7)$ & \\
\hline Biopsy site & & & $<0.001$ & & & $<0.001$ & & & 0.441 & & & 0.196 \\
\hline Liver & 38 & $1(2.6)$ & & 37 & $4(10.8)$ & & 38 & $14(36.8)$ & & 35 & $9(25.7)$ & \\
\hline Bone & 27 & $1(3.7)$ & & 26 & 7 (26.9) & & 26 & 7 (26.9) & & 26 & $4(15.4)$ & \\
\hline Lymph node & 31 & $11(35.5)$ & & 31 & $17(54.8)$ & & 30 & $13(43.3)$ & & 30 & $11(36.7)$ & \\
\hline Histological subtype & & & 0.123 & & & 0.098 & & & 0.449 & & & 0.896 \\
\hline Adenocarcinoma & 66 & $5(7.6)$ & & 64 & $15(23.4)$ & & 64 & 23 (35.9) & & 62 & $19(30.6)$ & \\
\hline Carcinoma & 13 & $3(23.1)$ & & 12 & 4 (33.3) & & 13 & $4(30.8)$ & & 12 & $2(16.7)$ & \\
\hline Squamous cell carcinoma & 8 & $3(37.5)$ & & 9 & $5(55.5)$ & & 9 & $6(66.7)$ & & 9 & 3 (33.3) & \\
\hline Poorly differentiated adenocarcinoma & 8 & $1(12.5)$ & & 8 & $2(25.0)$ & & 8 & $3(37.5)$ & & 7 & $2(28.6)$ & \\
\hline Poorly differentiated carcinoma & 15 & $2(13.3)$ & & 15 & $8(53.3)$ & & 15 & $5(33.3)$ & & 15 & $4(26.7)$ & \\
\hline PS (ECOG) & & & $0.457^{\star}$ & & & 0.457 & & & 0.208 & & & 0.193 \\
\hline $0-1$ & 47 & $6(12.8)$ & & 45 & $14(31.1)$ & & 45 & $19(42.2)$ & & 43 & $15(34.9)$ & \\
\hline$\geq 2$ & 34 & $2(5.9)$ & & 34 & $8(23.5)$ & & 35 & $10(28.6)$ & & 33 & $7(21.2)$ & \\
\hline
\end{tabular}

PS (ECOG), performance status (Eastern Cooperative Oncology Group) (22). *Fisher's exact test. Significant p-values are depicted in bold.

\section{DISCUSSION}

Our study demonstrated that the metabolism-related proteins MCT1, MCT4, CD147, GLUT1 and CAIX are expressed in an important portion of the CUP, indicating a hyperglycolytic phenotype. More importantly, we found evidence that this hyperglycolytic behavior is more frequently observed in the CUP located in lymph nodes. Unexpectedly, survival analysis of CUP patients demonstrated that positive expression of GLUT1 and CAIX, their co-expression with MCT4, and MCT1/CD147 co-expression were positively associated with improved survival.

The present study showed expressive plasma membrane expression frequencies of MCT1, MCT4, CD147, GLUT1 and CAIX but not MCT2 in the CUP. During the metabolic adaptation of cancer cells, the dual role of MCT1 and MCT4 in maintaining the glycolytic phenotype by, on the one hand, facilitating lactate efflux and, on the other hand, contributing to the preservation of intracellular $\mathrm{pH}$ through co-transport with a proton determines the increased expression of these isoforms in tumor cells (16), as well as that of their chaperone CD147 (17). Furthermore, GLUT1 facilitates glucose uptake, and CAIX, responsible for the reversible conversion of $\mathrm{CO}_{2}$ and regulation of intracellular $\mathrm{pH}$, contributes to the acid-mediated cancer cell invasive phenotype, both also contributing to the maintenance of the Warburg effect, particularly in oxygen-depleted (hypoxia) conditions $(28,29)$. By contrast, MCT2 expression was low, as expected, because MCT2, which is mostly involved in lactate uptake (30), is mainly found in the cytoplasm of tumor cells (31) and is associated with favorable prognostic characteristics in other types of cancers $(32,33)$. In accordance with the study by Kim and colleagues (20), using a series of 77 CUPs, the frequencies of MCT4 and GLUT1 observed were similar to ours, with MCT4 being present in $71.4 \%$ and GLUT1 in $46.8 \%$ of samples. However, the authors showed a lower expression frequency of CAIX (11.7\%) than our findings. The same study showed an association of GLUT1 expression with the histological subtype of metastasis, with squamous cell carcinomas presenting a higher frequency of expression of this protein. This result suggests a hyperglycolytic phenotype and agrees with our results, showing that MCT1 was more frequently expressed in squamous cell carcinomas, which may be related to aggressiveness, high grade, high proliferative activity and poor prognosis, as observed in other tumor types $(16,33,34)$. The study performed by Koo and collaborators (35), in which 69 CUPs were analyzed, also suggests a highly glycolytic profile that determines a worse prognosis in CUP and shows that histological subtypes have 


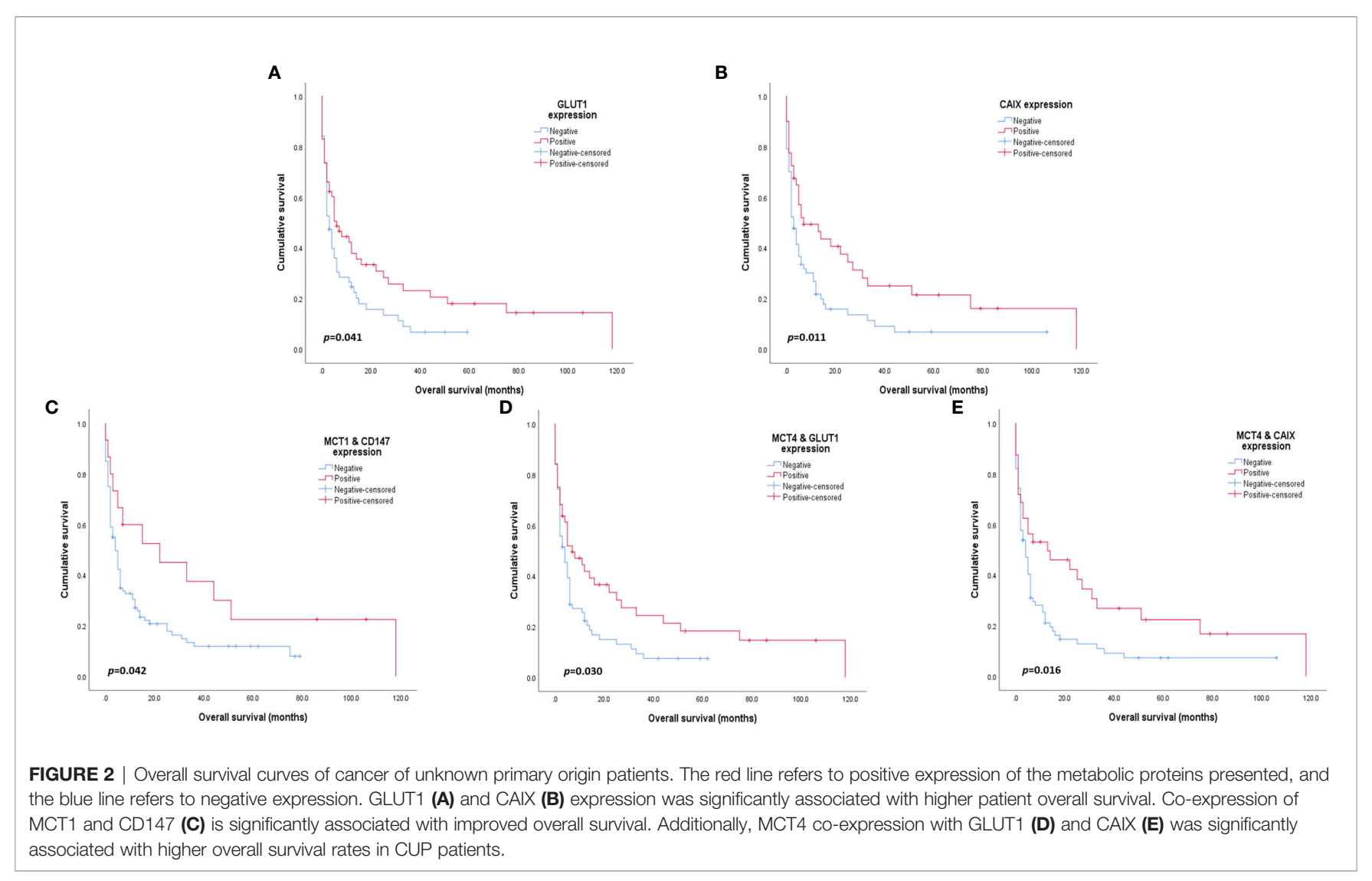

different metabolic behaviors. Among other proteins, the authors analyzed the expression of proteins involved in the metabolic reprogramming of cancer cells, such as HIF-1 $\alpha$, GLUT1, phosphorylated mTOR, phosphorylated S6, AMPK $\alpha 1$ and phosphorylated Akt, and identified a higher expression of proteins associated with the hypoxic tumor microenvironment, such as GLUT1 and HIF-1 $\alpha$, in squamous cell carcinomas. Additionally, GLUT1 and HIF-1 $\alpha$ expression was associated with a worse prognosis in this histological subtype, with HIF$1 \alpha$ being an independent factor for a worse prognosis (35). Thus, the feasibility of therapeutic approaches targeting metabolic reprogramming of CUP is suggested based on the evidence for metabolic heterogeneity of the different histological subtypes (20, 35). Additionally, MCT1 and CD147 were both more frequently expressed in samples from lymph nodes as well as cancers with lymph nodes as metastasis-dominant sites. A notable characteristic of the lymphatic system is its hypoxic environment, with the absence of red blood cell transport in lymphatic vessels. Generally, lymph vessels are located in remote oxygen-depleted areas, away from oxygen-carrying blood vessels. Tumor cells must adapt to this adverse hypoxic environment to sustain lymphangiogenesis and spread to lymph nodes, particularly with the upregulation of VEGF-C mediated by HIF-1 $\alpha$ activation (36).

In the search for a metabolic profile with clinical significance in CUP and a better understanding of this tumor entity, we evaluated co-expression between the proteins, and a significant association was found for MCT1/CD147, MCT4/CD147, MCT4/GLUT1 and
MCT4/CAIX co-expression. This finding suggests a hyperglycolytic and acid-resistant phenotype in a subset of CUPs, sustaining the Warburg effect for cell proliferation and cancer cell survival (11, 12, 37-40). MCT1/CD147 and MCT4/ CD147 co-expression was significantly associated with the lymph node biopsy site and the lymph node as the metastasis dominant site. These results were also found individually for MCT1 and CD147, supporting the synergism characteristic among MCT1, MCT4 and CD147 in the context of solid tumors related to microenvironment acidification and an increased metastatic potential $(34,41)$. The co-expression of MCTs with CD147 was previously described in colorectal tumor samples, including lymph node and hepatic metastasis, compared with adjacent normal tissues (42). The study highlights that MCT1/CD147 coexpression was observed in colorectal primary tumors, while MCT4/CD147 co-expression was observed in colorectal primary tumor, lymph node and hepatic metastasis samples (42). Thus, the co-expression findings of the present study are consistent with the biological role of these proteins because CD147 is a chaperone of MCT1 and MCT4 and is often co-expressed in tumor cells (42-44).

Unexpectedly, our results demonstrated that CUP patients with positive GLUT1 and CAIX expression presented significantly higher overall survival, and the same was observed for the coexpression of these proteins with MCT4 and MCT1/CD147 coexpression. Currently, most studies in the literature have shown that hyperglycolytic tumors present a poorer prognosis and decreased survival rates $(38,39,45,46)$. Conversely, our results 
TABLE 6 | Prognostic factors for the overall survival of patients with cancer of unknown primary origin.

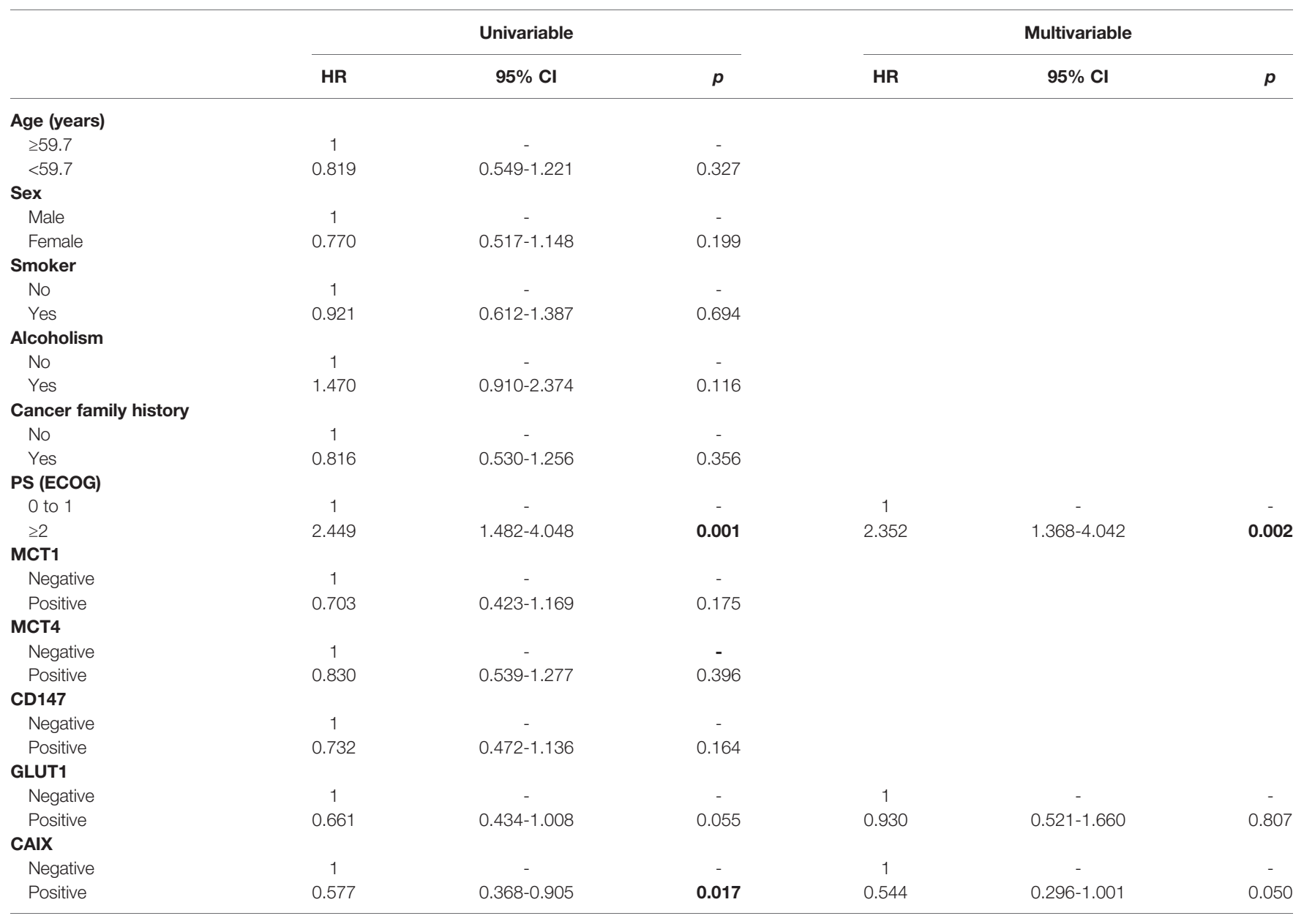

PS (ECOG), performance status (Eastern Cooperative Oncology Group) (22); HR, hazard ratio; Cl, confidence interval. Significant p-values are depicted in bold.

indicate a different behavior of this metabolic phenotype in CUP. First, we must consider that other isoforms of glucose transporters and carbonic anhydrases, such as GLUT3 and CAXII, may be present and active in these tumors, similar to other neoplasms (4753). However, because of sample limitations, we did not characterize the expression of these proteins in our samples, while studies considering the expression of GLUT3 and CAXII in CUP are lacking. Additionally, positive GLUT1 and CAIX expression does not influence the prognostic outcomes related to the overall and disease-free survival of cervical cancer patients (54). Cleven and co-workers (55) showed that positive GLUT1 expression was related to better survival in patients with colorectal tumors. Additionally, CAIX stromal and tumor expression was not related to clinical survival, with CAIX tumor expression showing decreased expression with advancing TNM and that earlier TNM stage has a more pronounced CAIX expression and better survival than later TNM stages. Furthermore, the authors demonstrated that stromal CAIX expression improved the survival rates of colorectal cancer patients, suggesting that tumor hypoxia may influence tumorassociated stromal cells that ultimately contributes to patient prognosis. Although the samples used in the present study were cancer treatment naïve, some studies demonstrated that systemic therapy alters the metabolic phenotype of cancer cells and affects the patient outcomes $(25,56,57)$. Goos and co-workers (56) showed that high GLUT1 expression levels in colorectal cancer liver metastasis were associated with improved survival of patients previously treated with systemic therapy. Additionally, a study performed by our group with locally advanced breast tumor patients submitted to neoadjuvant chemotherapy showed that CAIX-positive expression was associated with higher disease-free survival and disease-specific survival, and a pathological complete response after treatment (25), suggesting that neoadjuvant therapy affects the metabolic phenotype of aggressive glycolytic tumors, favoring the clinical outcomes of breast cancer patients. Regarding the association of MCT1/CD147, GLUT1/MCT4 and CAIX/ MCT4 co-expression with higher overall survival of CUP patients, to our best knowledge, this is the first report of MCT association with a good clinical outcome; however, it follows the rationale behind GLUT1 and CAIX findings, with glycolytic CUPs showing a better prognosis.

The main limitation of this study is related to the sample heterogeneity due to material from different biopsy sites as a result of metastases with different locations, influencing the analysis, 
particularly survival analysis. This situation leads to case loss, both during the selection of patients and immunohistochemical procedure due to the reduced tissue size and loss of representative tumor tissue perceived during immunohistochemical evaluation. The aforementioned impacted the total sample size and subgroup size, limiting the analysis considering the variables associated with the heterogeneous profile. Notably, the impossibility of comparing the metabolic profile of CUP samples with the normal tissue of origin is a limitation because the absence of a primary tumor defines this neoplastic entity. However, given the scarcity of previous studies characterizing cellular metabolism in CUP, this study represents an important gain for the literature.

In conclusion, although CUPs are biologically complex, our results suggest that a portion of these tumors present an increased expression of proteins related to glycolytic metabolism, with hyperglycolytic CUPs being associated with higher overall survival. New studies evaluating the metabolic profile of CUPs, as well as the characterization of other metabolism-related proteins and their activity, could contribute to a better understanding of this enigmatic entity of tumors, enhancing the clinical diagnosis, management and determination of new prognostic markers and therapeutic strategies.

\section{DATA AVAILABILITY STATEMENT}

The raw data supporting the conclusions of this article will be made available by the authors, without undue reservation.

\section{REFERENCES}

1. Massard C, Loriot Y, Fizazi K. Carcinomas of an Unknown Primary OriginDiagnosis and Treatment. Nat Rev Clin Oncol (2011) 8(12):701-10. doi: 10.1038/nrclinonc.2011.158

2. Rassy E, Pavlidis N. The Currently Declining Incidence of Cancer of Unknown Primary. Cancer Epidemiol (2019) 61:139-41. doi: 10.1016/ j.canep.2019.06.006

3. Rassy E, Pavlidis N. Progress in Refining the Clinical Management of Cancer of Unknown Primary in the Molecular Era. Nat Rev Clin Oncol (2020) 17:541-54. doi: 10.1038/s41571-020-0359-1

4. Stella GM, Senetta R, Cassenti A, Ronco M, Cassoni P. Cancers of Unknown Primary Origin: Current Perspectives and Future Therapeutic Strategies. J Transl Med (2012) 10:12. doi: 10.1186/1479-5876-10-12

5. van de Wouw AJ, Janssen-Heijnen ML, Coebergh JW, Hillen HF. Epidemiology of Unknown Primary Tumours; Incidence and PopulationBased Survival of 1285 Patients in Southeast Netherlands, 1984-1992. Eur J Cancer (2002) 38(3):409-13. doi: 10.1016/s0959-8049(01)00378-1

6. Bochtler T, Kramer A. Does Cancer of Unknown Primary (Cup) Truly Exist as a Distinct Cancer Entity? Front Oncol (2019) 9:402. doi: 10.3389/ fonc.2019.00402

7. Hanahan D, Weinberg RA. Hallmarks of Cancer: The Next Generation. Cell (2011) 144(5):646-74. doi: 10.1016/j.cell.2011.02.013

8. Warburg O, Wind F, Negelein E. The Metabolism of Tumors in the Body. J Gen Physiol (1927) 8(6):519-30. doi: 10.1085/jgp.8.6.519

9. Warburg O. On the Origin of Cancer Cells. Science (1956) 123(3191):309-14. doi: 10.1126/science.123.3191.309

10. Gatenby RA, Gillies RJ. Why Do Cancers Have High Aerobic Glycolysis? Nat Rev Cancer (2004) 4(11):891-9. doi: 10.1038/nrc1478

11. Vander Heiden MG, Cantley LC, Thompson CB. Understanding the Warburg Effect: The Metabolic Requirements of Cell Proliferation. Science (2009) 324 (5930):1029-33. doi: 10.1126/science.1160809

\section{ETHICS STATEMENT}

The studies involving human participants were reviewed and approved by the Barretos Cancer Hospital Ethics Committee. Written informed consent for participation was not required for this study in accordance with the national legislation and the institutional requirements.

\section{AUTHOR CONTRIBUTIONS}

$\mathrm{MB}, \mathrm{IF}, \mathrm{PB}$ and LP performed immunohistochemical reactions and wrote the manuscript. JJ, IF, PB and LP retrieved the clinicopathological data of patients from medical records. RC performed the statistical analysis. ES and AL-F analyzed the histological sections and performed the immunohistochemical evaluations. FC aided in the study design and discussion of the results. CP was responsible for the study design, contributed to the discussion of the results, organization and review of the manuscript. All authors contributed to the article and approved the submitted version.

\section{FUNDING}

This study was supported by FAPESP, Fundação de Amparo à Pesquisa do Estado de São Paulo (2016/08674-9; 2017/09226-2) and CNPq, Conselho Nacional de Desenvolvimento Científico e Tecnológico (306195/2016-0, 309998/2019-0).

12. Serpa J. Tumor Microenvironment: The Main Driver of Metabolic Adaptation. Switzerland: Springer International Publishing (2020). doi: 10.1007/978-3030-34025-4

13. Granja S, Pinheiro C, Reis RM, Martinho O, Baltazar F. Glucose Addiction in Cancer Therapy: Advances and Drawbacks. Curr Drug Metab (2015) 16 (3):221-42. doi: 10.2174/1389200216666150602145145

14. Macheda ML, Rogers S, Best JD. Molecular and Cellular Regulation of Glucose Transporter (GLUT) Proteins in Cancer. J Cell Physiol (2005) 202(3):654-62. doi: $10.1002 /$ jcp.20166

15. Medina RA, Owen GI. Glucose Transporters: Expression, Regulation and Cancer. Biol Res (2002) 35(1):9-26. doi: 10.4067/s0716-97602002000100004

16. Baltazar F, Pinheiro C, Morais-Santos F, Azevedo-Silva J, Queiros O, Preto A, et al. Monocarboxylate Transporters as Targets and Mediators in Cancer Therapy Response. Histol Histopathol (2014) 29(12):1511-24. doi: 10.14670/ $\mathrm{HH}-29.1511$

17. Xin X, Zeng X, Gu H, Li M, Tan H, Jin Z, et al. CD147/EMMPRIN Overexpression and Prognosis in Cancer: A Systematic Review and MetaAnalysis. Sci Rep (2016) 6:32804. doi: 10.1038/srep32804

18. Swietach P, Vaughan-Jones RD, Harris AL. Regulation of Tumor Ph and the Role of Carbonic Anhydrase 9. Cancer Metastasis Rev (2007) 26(2):299-310. doi: 10.1007/s10555-007-9064-0

19. Marchiq I, Pouyssegur J. Hypoxia, Cancer Metabolism and the Therapeutic Benefit of Targeting Lactate/H(+) Symporters. J Mol Med (Berl) (2016) 94 (2):155-71. doi: 10.1007/s00109-015-1307-x

20. Kim HM, Kim DH, Jung WH, Koo JS. Metabolic Phenotypes in Primary Unknown Metastatic Carcinoma. J Transl Med (2014) 12:2. doi: 10.1186/ 1479-5876-12-2

21. Luengo A, Gui DY, Vander Heiden MG. Targeting Metabolism for Cancer Therapy. Cell Chem Biol (2017) 24(9):1161-80. doi: 10.1016/j.chembiol.2017.08.028

22. Oken MM, Creech RH, Tormey DC, Horton J, Davis TE, McFadden ET, et al. Toxicity and Response Criteria of the Eastern Cooperative Oncology Group. Am J Clin Oncol (1982) 5(6):649-55. doi: 10.1097/00000421-198212000-00014 
23. Harris PA, Taylor R, Minor BL, Elliott V, Fernandez M, O'Neal L, et al. The REDCap Consortium: Building an International Community of Software Platform Partners. J BioMed Inform (2019) 95:103208. doi: 10.1016/j.jbi.2019.103208

24. Harris PA, Taylor R, Thielke R, Payne J, Gonzalez N, Conde JG. Research Electronic Data Capture (Redcap)-a Metadata-Driven Methodology and Workflow Process for Providing Translational Research Informatics Support. J BioMed Inform (2009) 42(2):377-81. doi: 10.1016/j.jbi.2008.08.010

25. Alves W, Bonatelli M, Dufloth R, Kerr LM, Carrara GFA, da Costa RFA, et al. CAIX is a Predictor of Pathological Complete Response and Is Associated With Higher Survival in Locally Advanced Breast Cancer Submitted to Neoadjuvant Chemotherapy. BMC Cancer (2019) 19(1):1173. doi: 10.1186/s12885-019-6353-2

26. Silva ECA, Carcano FM, Bonatelli M, Zaia MG, Morais-Santos F, Baltazar F, et al. The Clinicopathological Significance of Monocarboxylate Transporters in Testicular Germ Cell Tumors. Oncotarget (2018) 9(29):20386-98. doi: 10.18632/oncotarget.24910

27. Bonatelli M, Silva ECA, Carcano FM, Zaia MG, Lopes LF, Scapulatempo-Neto C, et al. The Warburg Effect Is Associated With Tumor Aggressiveness in Testicular Germ Cell Tumors. Front Endocrinol (Lausanne) (2019) 10:417. doi: 10.3389/fendo.2019.00417

28. Barron CC, Bilan PJ, Tsakiridis T, Tsiani E. Facilitative Glucose Transporters: Implications for Cancer Detection, Prognosis and Treatment. Metabolism (2016) 65(2):124-39. doi: 10.1016/j.metabol.2015.10.007

29. Becker HM. Carbonic Anhydrase IX and Acid Transport in Cancer. $\mathrm{Br} \mathrm{J}$ Cancer (2020) 122(2):157-67. doi: 10.1038/s41416-019-0642-Z

30. Payen VL, Mina E, Van Hee VF, Porporato PE, Sonveaux P. Monocarboxylate Transporters in Cancer. Mol Metab (2020) 33:48-66. doi: 10.1016/j.molmet.2019.07.006

31. Pinheiro C, Reis RM, Ricardo S, Longatto-Filho A, Schmitt F, Baltazar F. Expression of Monocarboxylate Transporters 1, 2, and 4 in Human Tumours and Their Association With CD147 and CD44. J BioMed Biotechnol (2010) 2010:427694. doi: 10.1155/2010/427694

32. Alves VA, Pinheiro C, Morais-Santos F, Felipe-Silva A, Longatto-Filho A, Baltazar F. Characterization of Monocarboxylate Transporter Activity in Hepatocellular Carcinoma. World J Gastroenterol (2014) 20(33):11780-7. doi: 10.3748/wjg.v20.i33.11780

33. Pinheiro C, Granja S, Longatto-Filho A, Faria AM, Fragoso MC, Lovisolo SM, et al. Metabolic Reprogramming: A New Relevant Pathway in Adult Adrenocortical Tumors. Oncotarget (2015) 6(42):44403-21. doi: 10.18632/oncotarget.5623

34. Le Floch R, Chiche J, Marchiq I, Naiken T, Ilc K, Murray CM, et al. CD147 Subunit of Lactate/H+ Symporters MCT1 and Hypoxia-Inducible MCT4 is Critical for Energetics and Growth of Glycolytic Tumors. Proc Natl Acad Sci USA (2011) 108(40):16663-8. doi: 10.1073/pnas.1106123108

35. Koo JS, Kim H. Hypoxia-Related Protein Expression and its Clinicopathologic Implication in Carcinoma of Unknown Primary. Tumour Biol (2011) 32 (5):893-904. doi: 10.1007/s13277-011-0190-5

36. Morfoisse F, Kuchnio A, Frainay C, Gomez-Brouchet A, Delisle MB, Marzi S, et al. Hypoxia Induces VEGF-C Expression in Metastatic Tumor Cells Via a HIF-1alpha-Independent Translation-Mediated Mechanism. Cell Rep (2014) 6(1):155-67. doi: 10.1016/j.celrep.2013.12.011

37. Bovenzi CD, Hamilton J, Tassone P, Johnson J, Cognetti DM, Luginbuhl A, et al. Prognostic Indications of Elevated MCT4 and CD147 Across Cancer Types: A Meta-Analysis. BioMed Res Int (2015) 2015:242437. doi: 10.1155/2015/242437

38. van Kuijk SJ, Yaromina A, Houben R, Niemans R, Lambin P, Dubois LJ. Prognostic Significance of Carbonic Anhydrase IX Expression in Cancer Patients: A Meta-Analysis. Front Oncol (2016) 6:69. doi: 10.3389/fonc.2016.00069

39. Wang J, Ye C, Chen C, Xiong H, Xie B, Zhou J, et al. Glucose Transporter GLUT1 Expression and Clinical Outcome in Solid Tumors: A Systematic Review and Meta-Analysis. Oncotarget (2017) 8(10):16875-86. doi: 10.18632/oncotarget.15171

40. Smallbone K, Gavaghan DJ, Gatenby RA, Maini PK. The Role of Acidity in Solid Tumour Growth and Invasion. J Theor Biol (2005) 235(4):476-84. doi: 10.1016/j.jtbi.2005.02.001

41. Kirk P, Wilson MC, Heddle C, Brown MH, Barclay AN, Halestrap AP. CD147 is Tightly Associated With Lactate Transporters MCT1 and MCT4 and Facilitates Their Cell Surface Expression. EMBO J (2000) 19(15):3896-904. doi: 10.1093/emboj/19.15.3896

42. Martins SF, Amorim R, Viana-Pereira M, Pinheiro C, Costa RF, Silva P, et al. Significance of Glycolytic Metabolism-Related Protein Expression in Colorectal Cancer, Lymph Node and Hepatic Metastasis. BMC Cancer (2016) 16:535. doi: 10.1186/s12885-016-2566-9
43. Semenza GL. Tumor Metabolism: Cancer Cells Give and Take Lactate. J Clin Invest (2008) 118(12):3835-7. doi: 10.1172/JCI37373

44. Wilson MC, Meredith D, Fox JE, Manoharan C, Davies AJ, Halestrap AP. Basigin (CD147) Is the Target for Organomercurial Inhibition of Monocarboxylate Transporter Isoforms 1 and 4: The Ancillary Protein for the Insensitive MCT2 Is EMBIGIN (Gp70). J Biol Chem (2005) 280 (29):27213-21. doi: 10.1074/jbc.M411950200

45. Javaeed A, Ghauri SK. MCT4 Has a Potential to be Used as a Prognostic Biomarker - A Systematic Review and Meta-Analysis. Oncol Rev (2019) 13 (2):403. doi: 10.4081/oncol.2019.403

46. Yu M, Chen S, Hong W, Gu Y, Huang B, Lin Y, et al. Prognostic Role of Glycolysis for Cancer Outcome: Evidence From 86 Studies. J Cancer Res Clin Oncol (2019) 145(4):967-99. doi: 10.1007/s00432-019-02847-w

47. Chen Z, Ai L, Mboge MY, Tu C, McKenna R, Brown KD, et al. Differential Expression and Function of CAIX and CAXII in Breast Cancer: A Comparison Between Tumorgraft Models and Cells. PloS One (2018) 13(7): e0199476. doi: 10.1371/journal.pone.0199476

48. Ivanov S, Liao SY, Ivanova A, Danilkovitch-Miagkova A, Tarasova N, Weirich G, et al. Expression of Hypoxia-Inducible Cell-Surface Transmembrane Carbonic Anhydrases in Human Cancer. Am J Pathol (2001) 158(3):905-19. doi: 10.1016/ S0002-9440(10)64038-2

49. Kocdor MA, Kocdor H, Pereira JS, Vanegas JE, Russo IH, Russo J. Progressive Increase of Glucose Transporter-3 (GLUT-3) Expression in Estrogen-Induced Breast Carcinogenesis. Clin Transl Oncol (2013) 15(1):55-64. doi: 10.1007/ s12094-012-0882-3

50. Krzeslak A, Wojcik-Krowiranda K, Forma E, Jozwiak P, Romanowicz H, Bienkiewicz A, et al. Expression of GLUT1 and GLUT3 Glucose Transporters in Endometrial and Breast Cancers. Pathol Oncol Res (2012) 18(3):721-8. doi: 10.1007/s12253-012-9500-5

51. Tafreshi NK, Bui MM, Bishop K, Lloyd MC, Enkemann SA, Lopez AS, et al. Noninvasive Detection of Breast Cancer Lymph Node Metastasis Using Carbonic Anhydrases IX and XII Targeted Imaging Probes. Clin Cancer Res (2012) 18(1):207-19. doi: 10.1158/1078-0432.CCR-11-0238

52. Watanabe M, Naraba H, Sakyo T, Kitagawa T. DNA Damage-Induced Modulation of GLUT3 Expression Is Mediated Through p53-Independent Extracellular Signal-Regulated Kinase Signaling in HeLa Cells. Mol Cancer Res (2010) 8(11):1547-57. doi: 10.1158/1541-7786.MCR-10-0011

53. Kuo MH, Chang WW, Yeh BW, Chu YS, Lee YC, Lee HT. Glucose Transporter 3 Is Essential for the Survival of Breast Cancer Cells in the Brain. Cells (2019) 8(12):1-14. doi: 10.3390/cells8121568

54. Kim BW, Cho H, Chung JY, Conway C, Ylaya K, Kim JH, et al. Prognostic Assessment of Hypoxia and Metabolic Markers in Cervical Cancer Using Automated Digital Image Analysis of Immunohistochemistry. J Transl Med (2013) 11:185. doi: 10.1186/1479-5876-11-185

55. Cleven AH, van Engeland M, Wouters BG, de Bruine AP. Stromal Expression of Hypoxia Regulated Proteins Is an Adverse Prognostic Factor in Colorectal Carcinomas. Cell Oncol (2007) 29(3):229-40. doi: 10.1155/2007/945802

56. Goos JA, de Cuba EM, Coupe VM, Diosdado B, Delis-Van Diemen PM, Karga C, et al. Glucose Transporter 1 (SLC2A1) and Vascular Endothelial Growth Factor A (Vegfa) Predict Survival After Resection of Colorectal Cancer Liver Metastasis. Ann Surg (2016) 263(1):138-45. doi: 10.1097/SLA.0000000 000001109

57. Euceda LR, Haukaas TH, Giskeødegård GF, Vettukattil R, Engel J, SilwalPandit L, et al. Evaluation of Metabolomic Changes During Neoadjuvant Chemotherapy Combined With Bevacizumab in Breast Cancer Using MR Spectroscopy. Metabolomics (2017) 13(4):37. doi: 10.1007/s11306-017-1168-0

Conflict of Interest: The authors declare that the research was conducted in the absence of any commercial or financial relationships that could be construed as a potential conflict of interest.

Copyright (๐ 2021 Bonatelli, Fornari, Bernécule, Pinheiro, Costa, Longatto-Filho, Junior, Silva, Cárcano and Pinheiro. This is an open-access article distributed under the terms of the Creative Commons Attribution License (CC BY). The use, distribution or reproduction in other forums is permitted, provided the original author(s) and the copyright owner(s) are credited and that the original publication in this journal is cited, in accordance with accepted academic practice. No use, distribution or reproduction is permitted which does not comply with these terms. 